\title{
BMJ Open Using provider-parent strategies to improve influenza vaccination in children and adolescents with special risk medical conditions: a randomised controlled trial protocol
}

\author{
Jane Tuckerman (D) , 1,2,3 Kelly Harper, ${ }^{4,5}$ Thomas R Sullivan (D) ,,7 \\ Jennifer Fereday, ${ }^{4}$ Jennifer Couper, ${ }^{1,4,5}$ Nicholas Smith, ${ }^{1,4}$ Andrew Tai, ${ }^{4,5}$ \\ Andrew Kelly, ${ }^{1,4}$ Richard Couper, ${ }^{4}$ Mark Friswell, ${ }^{4}$ Louise Flood, ${ }^{8}$ \\ Margaret Danchin, ${ }^{2,3,9}$ Christopher C Blyth, ${ }^{10,11,12,13}$ Helen Marshall (i) 1,4,5
}

To cite: Tuckerman J, Harper K, Sullivan TR, et al. Using provider-parent strategies to improve influenza vaccination in children and adolescents with special risk medical conditions: a randomised controlled trial protocol. BMJ Open 2022:12:e053838. doi:10.1136/ bmjopen-2021-053838

- Prepublication history for this paper is available online. To view these files, please visit the journal online (http://dx.doi org/10.1136/bmjopen-2021053838).

Received 26 May 2021 Accepted 27 December 2021

Check for updates

(C) Author(s) (or their employer(s)) 2022. Re-use permitted under CC BY-NC. No commercial re-use. See rights and permissions. Published by BMJ.

For numbered affiliations see end of article.

Correspondence to Dr Jane Tuckerman; jane.tuckerman@mcri.edu.au

\section{ABSTRACT}

Introduction Influenza immunisation is a highly cost-effective public health intervention. Despite a comprehensive National Immunisation Program, influenza vaccination in children and adolescents with special risk medical conditions (SRMCs) is suboptimal. Flutext- $4 \mathrm{U}$ is an innovative, multi-component strategy targeting paediatric hospitals, general practice and parents of children and adolescents with SRMC. The Flutext-4U study aims to assess the impact of Flutext-4U to increase influenza immunisation in children and adolescents with SRMC. Methods and analysis This is a randomised controlled trial involving parents of children and adolescents (aged $>6$ months to $<18$ years) with SRMC receiving tertiary care at the Women's and Children's Hospital (WCH), Adelaide, South Australia, who are eligible for funded influenza immunisation with a hospital appointment between the start of the seasonal influenza vaccination season and 31 July 2021, their treating general practitioners (GPS), and WCH paediatric specialists. Parents (of children/adolescents with SRMC) are randomised (1:1 ratio) to standard care plus intervention (SMS reminder messages to parents; reminders (written correspondence) for their child's GP from the hospital's Paediatric Outpatients Department) or standard care (hospital vaccine availability, ease of access and reminders for WCH subspecialists) with randomisation stratified by age-group ( $<5,5-14,>14$ to $<18$ years).

The primary outcome is influenza vaccination, as confirmed by the Australian Immunisation Register. The proportion vaccinated (primary outcome) will be compared between randomised groups using logistic regression, with adjustment made for age group at randomisation. The effect of treatment will be described using an $\mathrm{OR}$ with a $95 \% \mathrm{Cl}$.

Ethics and dissemination The protocol and all study materials have been reviewed and approved by the Women's and Children's Health Network Human Research Ethics Committee (HREC/20/WCHN/5). Results will be disseminated via peer-reviewed publication and at scientific meetings, professional and public forums.
Strengths and limitations of this study

- A randomised controlled trial will allow a determination of the impact of Flutext-4U intervention.

- This trial combines primary care and parent-level interventions and was designed for delivery in conjunction with a tertiary-level environment.

- The primary outcome is an objective measure, influenza vaccination receipt, which is confirmed on the Australian Immunisation Register.

- Standard care and intervention arms are independent but parent interaction particularly within subspecialties presents an inherent risk of contamination from intervention arm participants.

- To minimise bias at the tertiary provider level and because randomising subspecialists would be impractical and risk contamination, both arms will receive standard care.

Trial registration number Australian New Zealand Clinical Trials Registry (ACTRN12621000463875).

\section{INTRODUCTION}

In Australia, influenza is one of the most common vaccine preventable diseases, with direct healthcare costs estimated at $>\$ 115$ million per annum. ${ }^{12}$ Children and adolescents with special risk medical conditions, as defined in the Australian Immunisation Handbook (hereafter referred to as SRMCs) are a priority group for influenza immunisation, because of their significantly greater risk of influenza-associated hospitalisation and death. ${ }^{3-5}$ These conditions include: chronic heart, lung, neurological, metabolic, liver or kidney diseases; cancer; diabetes; Down syndrome and underlying immunosuppression. ${ }^{6}$ Around half of all children hospitalised 
with influenza in Australia have at least one $\mathrm{SRMC},{ }^{17}$ and these children are $30 \%-70 \%$ more likely to be admitted to intensive care, require mechanical ventilation, develop bacterial pneumonia, have prolonged hospitalisation or die following influenza infection. ${ }^{5}$

\section{Immunisation is the most effective strategy available to prevent influenza and its complications}

Individuals at highest risk of influenza-associated complications have been funded under the Australian National Immunisation Program, to receive the vaccine annually since $2010^{68}$; with the National Seasonal Influenza Vaccination Program generally commencing in the first month of autumn each year. The influenza vaccine in children can reduce the risk of influenza-associated hospitalisation by $65 \%-70 \%$, including children at increased risk. ${ }^{9} 10$ However, uptake is inadequate in children with SRMC, with coverage across Australia collectively across all SRMC only at $40 \%-52 \% .{ }^{11-13}$

\section{Barriers and facilitators to influenza immunisation}

Many reasons for low influenza immunisation rates in children with SRMC are modifiable and include: lack of awareness about recommendations, lack of information, not identifying children as being at risk, concern toward the vaccine/side effects, inconvenience, lack of perceived influenza severity, misinformation, negative social influences, need for a priming dose in children 6 months to 9 years, perceived low efficacy of the vaccine and vaccine access problems. ${ }^{12}{ }^{14-21}$ Conversely, children are more likely to receive the influenza vaccine if their parents recall the child's specialist recommending it, have adequate awareness and knowledge, believe that the vaccine is effective, safe and easy to access, and if their children are younger in age ( $<6$ years), have previously had influenza vaccine, have more than one SRMC, and that their parents or relatives believe it is necessary along with positive social influences. ${ }^{11} 131518-202223$

\section{Interventions to improve influenza immunisation coverage rates}

Data informing ways to overcome barriers to vaccine receipt are limited. A systematic review comprising 25 studies assessing strategies to improve influenza immunisation in children with SRMC found that interventions targeting practices, and parent or patients increased coverage by $15 \%$ (95\% CI $13 \%$ to $17 \%$ ) and $57 \%(52 \%$ to $61 \%$ ), respectively. ${ }^{24}$ However, most studies were conducted in the USA, focused only on children with asthma and used traditional reminder/recall systems (eg, written correspondence and telephone calls) that are financially costly, difficult to track receipt and labour intensive to administer. Text-message reminders sent by immunisation providers are a low-cost alternative and have been shown to increase vaccine uptake in some increased-risk groups. ${ }^{25-28}$ Notably no studies have investigated the impact of electronic reminders on influenza immunisation coverage in children with SRMC.
Approach to promoting influenza immunisation in children with SRMC

Despite a recommended and funded programme targeting children with SRMC, Australia lacks a coordinated implementation-coverage feedback loop, similar to other countries globally. ${ }^{29} 30$ While some hospitals have established services providing immunisation free of charge to children with SRMC, many hospitals and providers recommend that children attend their general practitioner (GP) for immunisation, adding to the burden of healthcare visits these families require. Research demonstrates that parents who receive a recommendation from their paediatrician or specialist are up to 16 times more likely to immunise their child. ${ }^{11}{ }^{13}$ However, less than $58 \%$ of parents recall their child's paediatrician recommending influenza immunisation when asked at the end of the season or the following year. ${ }^{11}{ }^{13}$ Current influenza immunisation protocols often lack consultation with providers and parents and vary significantly, even between departments within the same hospital. ${ }^{31}$

Multi-component interventions are optimal for improving immunisation coverage as they overcome many direct and indirect factors that affect the vaccine decisionmaking process to address multiple barriers simultaneously. Based in the USA, the Text4Health programme implements and evaluates, using randomised control trials, tailored, targeted vaccine text message reminders, with a focus on influenza in urban paediatric and pregnant populations. ${ }^{27} 283233$ Other research targeted at the diverse and complex information needs of pregnant women $^{34}{ }^{35}$ and other special-risk groups ${ }^{25}{ }^{26}$ demonstrate text-messaging interventions improve coverage. Barriers to influenza immunisation in children with SRMC include: (i) a lack of ready access to immunisation services; (ii) a lack of healthcare provider recommendation; (iii) providers advising against immunisation; (iv) safety concerns; (v) competing priorities; (vi) a lack of understanding of the need to immunise and (vii) being unaware of the recommendation to immunise. ${ }^{11-13} 36$ Several barriers also exist at the provider level with a sense of responsibility, knowledge and confidence of determining 'at risk' conditions, key drivers towards providing a recommendation. ${ }^{31}$

\section{Flutext-4U intervention}

The Flutext- $4 U$ intervention package includes three components which are centrally coordinated. At the tertiary-level: prompt/reminder stickers are placed on medical cases notes and bookmarks at the relevant clinical notes page for notes entry by the clinician to assist hospital specialists to facilitate vaccine recommendation; at the primary care-level: a hard copy communication letter is sent to the child's treating (referring) GP. The written correspondence will advise the GP of the quality improvement initiative to improve low rates of influenza in children with SRMCs and ask them to assist as part of the child's treating team to improve influenza vaccine uptake. The parent-level component includes a text 

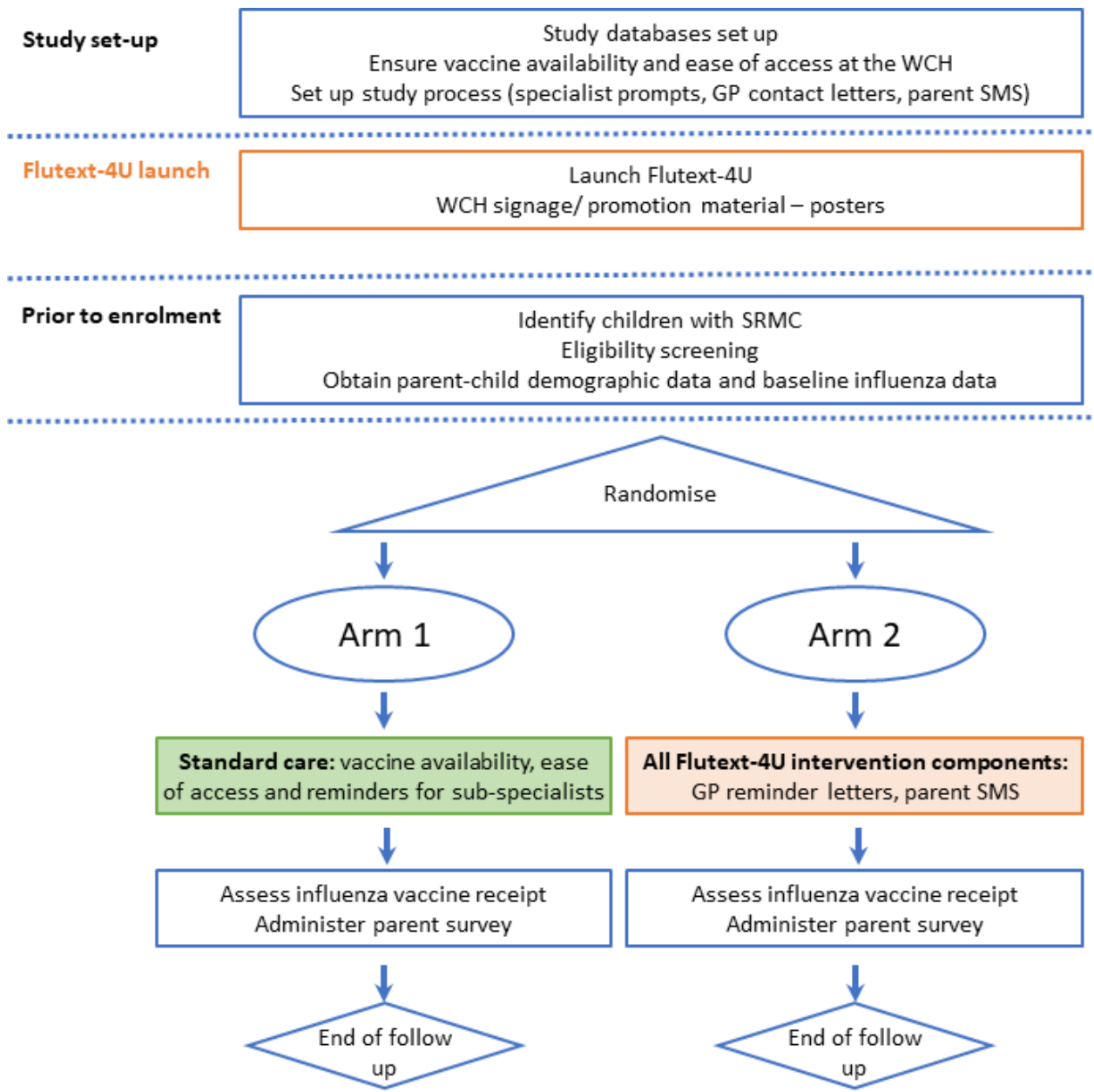

Figure 1 Study design. GP, general practitioner; SRMC, special risk medical condition; WCH, Women's and Children's Hospital.

message reminder sent to the child's parent (on behalf of the hospital) advising them that their child/adolescent is eligible for funded influenza vaccine and that they can receive it on request at the Women's and Children's Hospital (WCH) on-site immunisation clinic or at their GP.

\section{METHODS AND ANALYSIS Study design}

This parallel-group randomised controlled trial will measure the impact of the Flutext- $4 \mathrm{U}$ intervention on receipt of influenza vaccine in children with SRMC, who are patients attending specialist appointments at the WCH, South Australia. Participants will be randomly allocated to one of two combinations of Flutext- $4 \mathrm{U}$ components. Participants in study arm 1 (standard care) will receive the tertiary-level reminder prompts on medical case notes at outpatient appointments. Study arm 2 will comprise both the primary care and parent-level components, in addition to standard care (figure 1).

The WCH is one of four public hospitals across metropolitan Adelaide providing care to children and adolescents aged $<18$ years and is the state's leading provider of specialist care for children with acute and chronic conditions and the largest maternity and obstetric service. The WCH has 295 beds catering for all paediatric specialties and its Paediatric Emergency Department is a level 1 major trauma centre for children in South Australia. Each year, there are more than 30000 admissions and about 5000 births at the hospital. In addition, more than 250000 people come to the hospital as outpatients.

The study has been approved by the Women's and Children's Health Network Human Research Ethics Committee (HREC/20/WCHN/5). The study will be conducted in accordance with the Declaration of Helsinki and the International Conference on Harmonization Guidelines on Good Clinical Practice. Below, we describe the study protocol.

\section{Primary objective}

- Determine the difference in proportion of children and adolescents (aged $>6$ months to $<18$ years) in intervention vs standard treatment arm receiving at least one dose of influenza vaccine by 30 September 2021 (the end of the trial period), with receipt defined as receipt of one or more doses of influenza vaccine, confirmed on the Australian Immunisation Register (AIR) record (primary outcome) and parental report. 


\section{Secondary objectives}

- Determine the difference between intervention and standard treatment arms in the proportion of children and adolescents receiving at least one dose of influenza vaccine during the optimal period (1 April to 30 June).

- Determine the difference between intervention and standard treatment arms in the time from randomisation to vaccination.

- Determine whether the impact of the intervention on the primary outcome is modified by the subgroups: (i) age group $(<5 ; 5-14,>14$ to $<18$ years); (ii) residential location (metro or regional according to the predefined postcodes for metro and regional areas of South Australia) and (iii) paediatric subspecialty (diabetes, neurology, respiratory, gastroenterology, rheumatology, cardiology or other).

- Determine parental acceptability of the SMS intervention.

\section{Procedures}

\section{Randomisation}

Parents (of children/adolescents) will be randomised to study arm in a 1:1 ratio. The randomisation schedule will be prepared by an independent statistician (not otherwise involved in the conduct or analysis of the trial) using ralloc.ado version 3.7.6 in Stata V.16. Allocations will be performed using randomly permuted blocks, stratified by age-group $(<5,5-14,>14$ to $<18$ years $)$. The schedule will be provided electronically to the Women's and Children's Health Network (WCHN) ICT Applications System Support staff, who will allocate participants according to the randomisation schedule. The trial statistician will remain blinded.

\section{Study processes}

The Flutext-4U Study Coordinator will set up the system to deliver the specialist prompts (all study participants) and liaise with the WCHN ICT Applications System Support staff to set up text message reminders for parents and communication letters for GPs (trial arm \#2) to be sent centrally from the WCH. Influenza vaccine signage will be placed around hospital.

Children with SRMC will be identified from the WCH's Outpatient Department's appointment lists and eligibility screening completed with paediatric specialists on a fortnightly basis, using criteria set out in the National Immunisation Program for funded influenza vaccination. A waiver of consent was approved for parents to participate in this trial. Children will be ineligible if they have already received the 2021 influenza vaccine prior to trial commencement (defined as receipt on AIR); are a younger sibling of another trial participant (to ensure parents are not randomised twice); have no listed mobile phone number for parent/guardian; or have a diagnosis of Cystic Fibrosis, as these children already receive additional vaccine delivery support and influenza vaccine messaging within the WCH environment. Participants (parents) will be randomised to study arm and baseline demographic information will be collected for all parent-child pairs. Demographic data that may impact vaccine coverage will be collected and will include the child's age, gender, Aboriginal or Torres Strait Islander status, medical condition, postcode (to determine SocioEconomic Indexes for Areas (SEIFA) and residential location, ie, metro/regional) and previous influenza vaccine receipt in 2019 and 2020 (from AIR records).

Intervention components will be provided, as per study trial arm. Influenza vaccine reminder stickers and bookmarks will be placed on the hard copy paper medical case notes of all study participants, this will occur once eligibility and enrolment are confirmed and will be up to 2 weeks but no less than 1 week prior to the appointment. As per study arm, a communication (letter) with the child's treating (referring) GP will advise them that the child is identified as qualifying for funded influenza vaccine and seeking them to assist as part of the child's treating team to improve vaccine uptake. Parental SMS text message reminders will be sent using 'Message Media' software in a non-directive educational approach automatically to the child's parent advising them that their child/adolescent is eligible for funded influenza and where they can receive it. These will be timed to be sent prior to and following scheduled WCH specialist appointments and will be sent up to 2 weeks but no less than 1 week prior to the appointment. Each child will receive a maximum of three SMS reminders, for appointments scheduled between the start of the seasonal influenza vaccination season and the end of July. The second SMS will be sent 2 weeks after the first SMS and the third SMS sent 2 weeks after the second SMS. The first child will be enrolled on 15 April 2021. Text messages will cease if a parent replies to advise that the child is immunised and this is confirmed on the AIR. Text messages will comprise: (i) the influenza vaccination message reminder text, (ii) an option to reply if the vaccine has been received elsewhere. Parents will be encouraged to engage with their child's specialist, GP or immunisation provider to answer any related questions arising from the influenza vaccination message. Parents may opt-out of further text messages at any time.

At conclusion of the trial, parents in both trial arms will receive an SMS, to ask if the child had received an influenza vaccine in 2021. All collected identifiable data will be securely stored on a database held by the WCHN, with access to the database controlled by password protection. Any data presented will be de-identified prior to presentation.

\section{Patient and public involvement}

Patient and public involvement will include influenza vaccine signage designed in conjunction with the Flutext-4U Expert Advisory Group and WCH Consumer Advisory Committee. Parents in both trial arms will receive a parental acceptability survey. Study results will be communicated to key stakeholders and findings will 
also be disseminated in peer-reviewed scientific journals and presented at national and international conferences.

\section{Study monitoring and surveillance}

Flutext- $4 \mathrm{U}$ is a behavioural intervention and as such there are no risks or harms associated with drugs, procedures or devices in this study. The assessment of known potential risks and benefits of the intervention indicate negligible risk through participation in this study. Any risk of psychosocial distress associated with receiving a vaccine communication (SMS/letter/reminder) or discussing vaccines is unlikely and is outweighed by the anticipated benefits to the individual and or knowledge that might reasonably be expected from the results. The risk of the intervention is comparable to standard care. A risk assessment and management plan has been developed for all stages of the trial from trial design through to reporting and reflective of the nature of the trial as behavioural intervention. A Trial Management Group comprising the chief investigator, project manager and study coordinator and statistician will closely review all operational aspects of the conduct and progress of the trial and a Trial Steering Committee comprising the investigator team and specialist paediatricians from the study institution, will maintain clinical and ethical oversight.

\section{Sample size and analysis plan}

We plan to enrol at least 540 parents of children/adolescents medically at-risk receiving tertiary care at the WCH. In order to have $80 \%$ power to detect a $30 \%$ relative increase in the percentage of children vaccinated from $40 \%$ in the standard care arm to $52 \%$ in the trial arm containing all Flutext- $4 \mathrm{U}$ components, a sample size of 270 children per group is required (two-tailed alpha $=0.05$ ). Previous studies have shown a $30 \%-70 \%$ relative increase following other immunisation interventions. A 30\% relative increase in the percentage vaccinated would be considered clinically meaningful.

All analyses will be undertaken on an intention-to-treat basis according to a statistical analysis plan, prespecified prior to database lock. For the primary outcome, the number and proportion of participants receiving influenza vaccination in each group will be compared between randomised groups using logistic regression, with adjustment made for age-group $(<5,5-14,>14$ years $)$. The effect of treatment will be described using an OR with a 95\% CI. Subgroup analysis will examine the effect of paediatric subspecialty (diabetes, neurology, respiratory, gastroenterology, rheumatology, cardiology or other), age group $(<5,5-14,>14$ to $<18$ years $)$ and residential location (metropolitan, rural according to the predefined postcodes for metropolitan and regional areas of South Australia) on the primary outcome. Secondary analyses will be performed using logistic regression for binary outcomes and a Cox proportional hazards model for time to event outcomes, again with adjustment for age group $(<5,5-14,>14$ to $<18$ years $)$. In all analyses, a two-sided $p$ value $<0.05$ will be used to indicate statistical significance.
No adjustment will be made for multiple preplanned comparisons, as the overall comparison of vaccine uptake is of primary interest. The study statistician undertaking analysis will remain blinded to trial intervention assignment.

\section{DISCUSSION}

This study will assess the effectiveness of a structured multimodal strategy using evidence-based tools and targeting a paediatric hospital and parents of children with SRMC to increase child influenza immunisation coverage rates. The intervention combines primary carelevel and parent-level interventions and was designed for delivery in conjunction with a tertiary-level environment.

Improving immunisation coverage in high-risk populations remains at the forefront of implementation research. Yet, investment into programmes and research to ensure funded vaccines are administered to those who need them most has been limited. Only $43.9 \%$ (WCH) children with SRMC received influenza vaccine in 2015, despite a funded influenza programme for this at-risk group. $^{11}$

Many modifiable barriers to annual influenza immunisation exist and multimodal strategies using: (i) practicelevel or (ii) patient (or parent)-level interventions have been shown to improve immunisation rates. Employing extensive pilot data obtained by our research from parents and healthcare workers we are uniquely placed to develop, implement and evaluate Flutext-4U. Flutext- $4 \mathrm{U}$ is a structured multimodal strategy using evidence-based tools and targeting paediatric hospitals and parents of children with SRMC to increase child influenza immunisation coverage rates. Flutext-4U will be implemented at the WCH using a randomised controlled trial design followed by thorough evaluation.

We are mindful of the inherent risk from contamination from intervention arm participants, particularly within medical risk groups due to parent interaction. To minimise bias at the tertiary provider level and because randomising subspecialists would be impractical, with an almost certain chance of cross-contamination, both intervention arms will receive standard care.

It is also important to collect data on parental acceptability of the intervention as this will have the potential to inform any adaptations to the future implementation of the intervention. Flutext- $4 \mathrm{U}$ will develop coordinated approaches to immunising children with SRMC and establish the evidence required to optimise paediatric influenza immunisation strategies and campaigns. It will provide a model for future targeted high-risk programmes nationally.

If the study demonstrates no negative effects, the intervention will be subsequently implemented for all children with SRMC at the WCH. Any impact on coverage will assist other Australian jurisdictions and national programme directors in the implementation of similar programmes. Additionally, a focus on developing low-cost, adaptable 
and scalable methods for improving coverage rates is expected to have implications for many at-risk populations so that the intervention could be adapted and tested in other populations.

\section{Ethics and dissemination}

The protocol and all study materials have been reviewed and approved by the Women's and Children's Health Network Human Research Ethics Committee (HREC/20/ WCHN/5). The trial will be conducted in compliance with the current version of the protocol. Any change to the protocol document that affects the scientific intent, trial design, participant safety or may affect a participant's willingness to continue participation in the trial is considered an amendment, and will be submitted to the HREC, for approval prior to being implemented. Following completion of the trial, the results will be disseminated via peer-reviewed publication and at scientific meetings, professional and public forums. The results will be disseminated regardless of the magnitude or direction of effect. Authorship will be allocated using the guidelines for authorship defined by the International Committees of Medical Journal Editors and the role of each author will be published in line with journal requirements. There are no plans for the use of professional writers.

\section{Author affiliations \\ ${ }^{1}$ Adelaide Medical School, The University of Adelaide, Adelaide, South Australia, Australia \\ ${ }^{2}$ Vaccine Uptake Group, Murdoch Children's Research Institute, Melbourne, Victoria, Australia \\ ${ }^{3}$ Faculty of Medicine, Dentistry and Health Sciences, University of Melbourne, Melbourne, Victoria, Australia \\ ${ }^{4}$ Women's and Children's Health Network, North Adelaide, South Australia, Australia ${ }^{5}$ Robinson Research Institute, The University of Adelaide, Adelaide, South Australia, Australia \\ ${ }^{6}$ SAHMRI Women and Kids, South Australian Health and Medical Research Institute, Adelaide, South Australia, Australia \\ ${ }^{7}$ School of Public Health, The University of Adelaide, Adelaide, South Australia, Australia \\ ${ }^{8}$ Communicable Disease Control Branch, South Australian Department of Health and Wellbeing, Adelaide, South Australia, Australia \\ ${ }^{9}$ Department of General Medicine, The Royal Children's Hospital, Melbourne, Victoria, Australia \\ ${ }^{10}$ Wesfarmers Centre of Vaccines and Infectious Diseases, Telethon Kids Institute, University Western Australia, Perth, Western Australia, Australia \\ ${ }^{11}$ Department of Infectious Diseases, Perth Children's Hospital, Perth, Western Australia, Australia \\ ${ }^{12}$ School of Medicine, University of Western Australia, Perth, Western Australia, Australia \\ ${ }^{13}$ Department of Microbiology, PathWest Laboratory Medicine WA, Perth, Western Australia, Australia}

Contributors JT wrote the first draft with assistance from KH, TRS and HM. JF, JC, $\mathrm{NS}, \mathrm{AT}, \mathrm{AK}, \mathrm{RC}, \mathrm{MF}, \mathrm{LF}, \mathrm{MD}$ and CCB contributed to the manuscript and all authors approved the final version for publication.

Funding This work is supported by a Women's and Children's Hospital Foundation Grant.

Competing interests JT is an investigator on a project grant sponsored by Industry. Her institution has received funding from Industry (GSK) for investigator led research. She does not receive any personal payments from Industry. HM is an investigator on clinical vaccine trials sponsored by Industry (Pfizer, GSK). Her institution receives funding for Investigator led research from GSK, Pfizer, Sanofi-
Pasteur. All other authors (KH, TRS, JF, JC, NS, AT, AK, RC, MF, LF, MD, CCB) report no conflicts of interest.

Patient and public involvement Patients and/or the public were involved in the design, or conduct, or reporting, or dissemination plans of this research. Refer to the Methods section for further details.

Patient consent for publication Not applicable.

Provenance and peer review Not commissioned; externally peer reviewed.

Open access This is an open access article distributed in accordance with the Creative Commons Attribution Non Commercial (CC BY-NC 4.0) license, which permits others to distribute, remix, adapt, build upon this work non-commercially, and license their derivative works on different terms, provided the original work is properly cited, appropriate credit is given, any changes made indicated, and the use is non-commercial. See: http://creativecommons.org/licenses/by-nc/4.0/.

\section{ORCID iDs}

Jane Tuckerman http://orcid.org/0000-0001-6938-1751

Thomas R Sullivan http://orcid.org/0000-0002-6930-5406

Helen Marshall http://orcid.org/0000-0003-2521-5166

\section{REFERENCES}

1 Blyth CC, Macartney KK, McRae J, et al. Influenza epidemiology, vaccine coverage and vaccine effectiveness in children admitted to sentinel Australian hospitals in 2017: results from the PAEDS-FluCAN collaboration. Clin Infect Dis 2019;68:940-8.

2 Newall AT, Scuffham PA. Influenza-related disease: the cost to the Australian healthcare system. Vaccine 2008;26:6818-23.

3 World Health Organisation. Vaccines against influenza who position paper - November 2012. Wkly Epidemiol Rec 2012;87:461-76.

4 Gill PJ, Ashdown HF, Wang K, et al. Identification of children at risk of influenza-related complications in primary and ambulatory care: a systematic review and meta-analysis. Lancet Respir Med 2015;3:139-49.

5 Tuckerman J, Misan S, Crawford NW, et al. Influenza in children with special risk medical conditions: a systematic review and metaanalysis. Pediatr Infect Dis J 2019;38:912-9.

6 Australian Technical Advisory Group on Immunisation (ATAGI). The Australian Immunisation Handbook [Electronic book]. Canberra: Australian Government Department of Health, 2018. Available: https://immunisationhandbook.health.gov.au/

7 Lakhan N, Clarke M, Mathew SM, et al. Retrospective review of factors associated with severe hospitalised community-acquired influenza in a tertiary paediatric hospital in South Australia. Influenza Other Respir Viruses 2016;10:479-85.

8 Australian Technical Advisory Group on Immunisation (ATAGI). Statement on the administration of seasonal influenza vaccines in 2019. Canberra: Australian government department of health; 5 April, 2019. Available: https://beta.health.gov.au/file/10519/download? token=1zBZrm6E

9 Blyth CC, Jacoby P, Effler PV, et al. Effectiveness of trivalent flu vaccine in healthy young children. Pediatrics 2014;133:e1218-25.

10 Blyth CC, Jacoby P, Effler PV, et al. Influenza vaccine effectiveness and uptake in children at risk of severe disease. Pediatr Infect Dis J 2016;35:309-15.

11 Tuckerman J, Misan S, Salih S, et al. Influenza vaccination: uptake and associations in a cross-sectional study of children with special risk medical conditions. Vaccine 2018;36:8138-47.

12 Newcombe J, Kaur R, Wood N, et al. Prevalence and determinants of influenza vaccine coverage at tertiary pediatric hospitals. Vaccine 2014;32:6364-8.

13 Norman DA, Danchin M, Van Buynder P, et al. Caregiver's attitudes, beliefs, and experiences for influenza vaccination in Australian children with medical comorbidities. Vaccine 2019;37:2244-8.

14 Pandolfi E, Marino MG, Carloni E, et al. The effect of physician's recommendation on seasonal influenza immunization in children with chronic diseases. BMC Public Health 2012;12:984.

15 Esposito S, Marchisio P, Droghetti R, et al. Influenza vaccination coverage among children with high-risk medical conditions. Vaccine 2006;24:5251-5.

16 Daley MF, Beaty BL, Barrow J, et al. Missed opportunities for influenza vaccination in children with chronic medical conditions. Arch Pediatr Adolesc Med 2005;159:986-91.

17 Romano M, Pandolfi E, Marino MG, et al. Seasonal and pandemic influenza vaccine: recommendations to families of at-risk children during the 2009-10 season. Eur J Public Health 2012;22:821-4. 
18 Gnanasekaran SK, Finkelstein JA, Hohman K, et al. Parental perspectives on influenza vaccination among children with asthma. Public Health Rep 2006;121:181-8.

19 Lin CJ, Zimmerman RK, Nowalk MP, et al. Parental perspectives on influenza vaccination of children with chronic medical conditions. $J$ Natl Med Assoc 2006;98:148-53.

20 Peleg N, Zevit N, Shamir R, et al. Seasonal influenza vaccination rates and reasons for non-vaccination in children with gastrointestinal disorders. Vaccine 2015;33:182-6.

21 Principi N, Esposito S. Pediatric influenza prevention and control. Emerg Infect Dis 2004;10:574-80.

22 Lin CJ, Nowalk MP, Zimmerman RK, et al. Beliefs and attitudes about influenza immunization among parents of children with chronic medical conditions over a two-year period. J Urban Health 2006;83:874-83.

23 Rodríguez-Rieiro C, Domínguez-Berjón MF, Esteban-Vasallo MD, et al. Vaccination coverage against 2009 seasonal influenza in chronically ill children and adults: analysis of population registries in primary care in Madrid (Spain). Vaccine 2010;28:6203-9.

24 Norman DA, Barnes R, Pavlos R, et al. Improving influenza vaccination in children with comorbidities: a systematic review. Pediatrics 2021;147. doi:10.1542/peds.2020-1433. [Epub ahead of print: 0802 2021].

25 Stephens AB, Wynn CS, Stockwell MS. Understanding the use of digital technology to promote human papillomavirus vaccination - A RE-AIM framework approach. Hum Vaccin Immunother 2019;15:1549-61.

26 Regan AK, Bloomfield L, Peters I, et al. Randomized controlled trial of text message reminders for increasing influenza vaccination. Ann Fam Med 2017;15:507-14.

27 Stockwell MS, Kharbanda EO, Martinez RA, et al. Effect of a text messaging intervention on influenza vaccination in an urban, low-income pediatric and adolescent population: a randomized controlled trial. JAMA 2012;307:1702-8.
28 Stockwell MS, Hofstetter AM, DuRivage N, et al. Text message reminders for second dose of influenza vaccine: a randomized controlled trial. Pediatrics 2015;135:e83-91.

29 European Centre for Disease Prevention and Control. Seasonal influenza vaccination in Europe. vaccination recommendations and coverage rates in the EU member states for eight influenza seasons: 2007-2008 to 2014-2015. Stockholm: ECDC, 2017.

30 European Centre for Disease Prevention and Control. Seasonal influenza vaccination in Europe - Overview of vaccination recommendations and coverage rates in the EU Member States for the 2012-13 influenza season. Stockholm: ECDC, 2015.

31 Tuckerman J, Crawford NW, Marshall HS. Seasonal influenza vaccination for children with special risk medical conditions: does policy meet practice? J Paediatr Child Health 2020;56:1388-95.

32 Stockwell MS, Kharbanda EO, Martinez RA, et al. Text4Health: impact of text message reminder-recalls for pediatric and adolescent immunizations. Am J Public Health 2012;102:e15-21.

33 Hofstetter AM, Vargas CY, Kennedy A, et al. Parental and provider preferences and concerns regarding text message reminder/recall for early childhood vaccinations. Prev Med 2013;57:75-80.

34 Chamberlain AT, Seib K, Ault KA, et al. Improving influenza and Tdap vaccination during pregnancy: a cluster-randomized trial of a multicomponent antenatal vaccine promotion package in late influenza season. Vaccine 2015;33:3571-9.

35 Kaufman J, Attwell K, Tuckerman J, et al. Feasibility and acceptability of the multi-component P3-MumBubVax antenatal intervention to promote maternal and childhood vaccination: a pilot study. Vaccine 2020;38:4024-31.

36 P Newcombe J, Kaur R, Wood N, et al. Paediatrician beliefs and practices around influenza vaccination. J Paediatr Child Health 2017;53:711-4 